\title{
All but one free ride when wealth effects are small
}

\author{
Joaquim Silvestre
}

Received: 15 November 2010 / Accepted: 31 March 2011 / Published online: 19 April 2011

(C) The Author(s) 2011. This article is published with open access at SpringerLink.com

\begin{abstract}
Quasilinear preferences on a public good and a numeraire good are limits of preferences where both goods are normal. The set of equilibria of the voluntary contribution (or private provision) game is easily characterized under quasilinearity by: top valuators aggregately contribute their common stand-alone contribution, whereas non-top valuators contribute nothing. Because, as long as preferences are randomly selected, there will typically be a single top valuator, it follows that, typically, the equilibrium is unique, with all players but one contributing nothing, hence "free riding" in the sense of the ordinary English usage of the expression. The upper-hemicontinuity of the Nash equilibrium correspondence implies that this is also the case when both goods are strictly normal, but the wealth effects on the public good are small.
\end{abstract}

Keywords Free riding - Public goods - Voluntary contributions · Private provision · Normal goods · Quasilinear preferences · Wealth effects

JEL Classification $\quad \mathrm{H} 41 \cdot \mathrm{C} 72 \cdot \mathrm{D} 70$

\section{Free riding}

The terms free rider and free riding are frequently used both in economics and in ordinary English speech. "Free rider" has been present in economics at least since Buchanan (1964) with the following meaning: the free-rider problem is defined as the inefficiency of the outcome of a scheme to supply a public good by voluntary

I am indebted to the referees and editor of the Review for helpful comments.

\footnotetext{
J. Silvestre ( $\square)$

University of California, Davis, USA

e-mail: jbsilvestre@ucdavis.edu
} 
contribution. Hence, the free rider problem is particular case of the inefficiency of laissez-faire in the presence of externalities. A "free rider" in this context is somebody who does not take account of the effects of her actions on other people. Note that every participant in the usual game of voluntary contributions to public goods is a free rider in this sense, because even if a person does contribute a positive amount, her contribution would be larger if she took into account the positive externality that she generates. Because inefficiency is bad, "free riding" has a clear negative connotation in the economics discourse.

The meaning is somewhat different in the common English usage of the expression: a "free rider" is somebody who benefits from something without paying. The Oxford English Dictionary attests the term "ride" from 1886 the sense of a "rider" as "A passenger, esp. one using public transport." "Free rider" appears in the legal literature at the turn of the 19th century in reference to not-paying passengers in trains, both with and without the authorization of the railroad, and in the 1930's for hitchhikers. The term did not necessarily have a negative connotation in its earlier usage: it could perfectly apply to a VIP who was a guest of the railroad company. ${ }^{1}$

Here I use "free rider" in the sense of the ordinary English usage, i.e., as a person who contributes nothing. Granted, the discussion is framed by the voluntary contribution game where the equilibrium is indeed inefficient. But my focus is the characterization of who pays and who does not, rather than efficiency.

\section{The model}

Let there be a finite number of players, numbered $i=1, \ldots, I$, and let $I$ also denote the set of players. For $i=1, \ldots, I$, Player $i$ 's utility function is denoted $u_{i}: \Re_{+} \times M_{i} \rightarrow$ $\Re:\left(y, m_{i}\right), \mapsto u_{i}\left(y, m_{i}\right)$, where $M_{i} \subset \Re, y$ is the amount of the public good, and $m_{i} \in M_{i}$ is the amount of a private, numeraire good. Player $i$ is endowed with $\omega_{i}$ units of numeraire, and decides on her contribution $t_{i}$ towards the supply of the public good. We require $t_{i}$ to be nonnegative. Given a tuple $\left(t_{1}^{*}, \ldots, t_{I}^{*}\right)$, we define the supply of the public good as $t^{*} \equiv \sum_{h \in I} t_{h}^{*}$ and, for $i \in I$, we write $t_{-i}^{*} \equiv \sum_{\substack{h \in I \\ h \neq i}} t_{h}^{*}$.

A Nash equilibrium of the voluntary contribution (or private provision) game is a tuple $\left(t_{1}^{*}, \ldots, t_{I}^{*}\right) \in \Re_{+}^{I}$ of contributions such that, for each $i \in I, t_{i}^{*}$ maximizes $u_{i}\left(t_{-i}^{*}+t_{i}, \omega_{i}-t_{i}\right)$ subject to $t_{i} \geq 0$.

Let $\left(t_{1}^{*}, \ldots, t_{I}^{*}\right)$ be an equilibrium. We say that $i$ is a contributor at $\left(t_{1}^{*}, \ldots, t_{I}^{*}\right)$ if $t_{i}^{*}>0$. If, on the contrary, $t_{i}^{*}=0$, then we say that, at $\left(t_{1}^{*}, \ldots, t_{I}^{*}\right), i$ is a non contributor or that $i$ free rides.

\section{Normality}

It is well known that when both the public good and the private good are normal (positive wealth effects) the following properties hold (Warr 1983; Bergstrom et al. 1986, 1992; Andreoni 1988; Fraser 1992; Buchholz et al. 2006a,b).

\footnotetext{
${ }^{1}$ See Silvestre (2008), where the difficulties in translating the "free rider" into other languages are discussed.
} 
(1) Uniqueness (Bergstrom et al. 1986, Theorems 2-3): There is a unique Nash equilibrium, which implies a unique tuple of consumption vectors.

(2) Warr neutrality (Warr 1983; Bergstrom et al. 1986): A redistribution of wealth among contributors does not change the equilibrium consumption vectors.

\section{Quasilinearity}

\subsection{Assumptions}

Quasilinearity assumption. $M_{i}=\Re$ and $u_{i}\left(y, m_{i}\right)=v_{i}(y)+m_{i}$, for some function $v_{i}: \Re_{+} \rightarrow \Re$.

Remark 1 Postulating $M_{i}=\Re$ guarantees the global absence of wealth effects (see, e.g., Mas-Colell et al. 1995). Alternatively, as it is frequently done, one can assume that $M_{i}=\Re_{+}$and that $\omega_{i}$ is "high enough," which here requires $\omega_{i}$ to exceed $i$ 's "stand-alone contribution," defined below.

We may variously refer to $v_{i}(y)$ as $i$ 's valuation of, benefit from, or willingness to pay for $y$ units of the public good. Accordingly, when $v_{i}(y)$ is differentiable, $v_{i} \prime(y)$ is the marginal valuation of (or benefit from, or willingness to pay for) the public good at $y$. Note that if the free disposal of the public good is postulated, then $v_{i}$ cannot be decreasing anywhere.

Single peakedness assumption. The function $b_{i}: \Re_{+} \rightarrow \Re: b_{i}(y)=v_{i}(y)-y$ is single peaked, i.e., $\exists \hat{y}_{i} \geq 0$ such that

$$
\begin{array}{ll}
b_{i}\left(\hat{y}_{i}\right)>b_{i}(y) & \text { for all } y \geq 0, \\
\text { if } y^{0}<y^{1}<\hat{y}_{i}, & \text { then } b_{i}\left(y^{1}\right)>b_{i}\left(y^{0}\right), \\
\text { if } \hat{y}_{i}<y^{0}<y^{1}, & \text { then } b_{i}\left(y^{0}\right)>b_{i}\left(y^{1}\right) .
\end{array}
$$

Under the single-peakedness assumption we call $\hat{y}_{i} i$ 's stand-alone contribution.

Note that the single peakedness assumption holds if and only if $b_{i}$ is strictly quasiconcave and has a maximizer.

The single-peakedness assumption is satisfied by most, if not all, valuation functions used in the modeling of the private provision game. It is in particular satisfied in the popular case where $v_{i}$ is strictly concave, continuous at $y=0$ and bounded. It is also satisfied when $v_{i}$ is continuous, increasing and strictly concave on an interval $[0, \bar{y}]$ and constant on $(\bar{y}, \infty):$ the amount $\bar{y}$ is then a satiation level of the public good, and a free disposal postulate makes the valuation function constant above $\bar{y}$.

Given an profile of $I$ quasilinear preferences, define the set of top valuators as $T \equiv\left\{i \in I: \hat{y}_{i} \geq \hat{y}_{h}, \forall h \in I\right\}$, and the top valuation as $\hat{y} \equiv \hat{y}_{i}$, for any $i \in T$. Quasilinearity implies that whether $i$ is a top valuator or not depends only on the profile of preferences, and not on the vector of individual endowments of the numeraire good.

\subsection{Characterization of the Nash equilibrium}

Proposition Under quasilinearity and single peakedness $\left(t_{1}^{*}, \ldots, t_{1}^{*}\right)$ is a Nash equilibrium if and only if it is nonnegative and satisfies 
(a) $t^{*}=\hat{y}$;

(b) $i \notin T \Rightarrow t_{i}^{*}=0$.

Proof (Straightforward; offered for self-containment.) We first show that (a) and (b) imply that $\left(t_{1}^{*}, \ldots, t_{1}^{*}\right)$ is a Nash equilibrium. Suppose not, i.e., there is a player $i$ and a $t_{i} \geq 0$ such that $v_{i}\left(t_{-i}^{*}+t_{i}\right)+\omega_{i}-t_{i}>v_{i}\left(t^{*}\right)+\omega_{i}-t_{i}^{*}$, i.e.,

$$
v_{i}\left(t_{-i}^{*}+t_{i}\right)+\omega_{i}-t_{-i}^{*}-t_{i}>v_{i}\left(t^{*}\right)+\omega_{i}-t_{-i}^{*}-t_{i}^{*} .
$$

Because, by (a), $t^{*}$ is the stand-alone contribution of a top valuator, (1) cannot hold for $i \in T$.

So let $i \notin T$, which implies that $i$ /s stand-alone's contribution is strictly less than the stand-alone contribution of a top valuator, i.e., $\hat{y}_{i}<\hat{y}=t^{*}=t_{-i}^{*}$ (by (a) and (b)). Therefore $\hat{y}_{i}<t_{-i}^{*} \leq t_{-i}^{*}+t^{\prime}$, yet $b_{i}\left(t_{-i}^{*}+t_{i}\right)>b_{i}\left(t_{-i}^{*}\right)$ (by (1)), contradicting the single peakedness of $b_{i}$.

Conversely, let $\left(t_{1}^{*}, \ldots, t_{1}^{*}\right)$ be a Nash equilibrium. To prove (a), assume, by contradiction, that $t^{*} \neq \hat{y}$. If $t^{*}<\hat{y}$, then choose a top valuator $i$, in which case $v_{i}(\hat{y})-$ $\hat{y}>v_{i}\left(t^{*}\right)-t^{*}$, i.e., $v_{i}\left(t_{-i}^{*}+\hat{y}-t_{-i}^{*}\right)+t_{-i}^{*}-\hat{y}>v_{i}\left(t_{-i}^{*}+t_{i}^{*}\right)+t_{-i}^{*}-t_{i}^{*}$, or writing $t_{i} \equiv \hat{y}-t_{-i}^{*}$, we have that $t_{i}>0$ (because $\hat{y}>t^{*} \geq t_{-i}^{*}$ ) and that $v_{i}\left(t_{-i}^{*}+t_{i}\right)-t_{i}>v_{i}\left(t_{-i}^{*}+t_{i}^{*}\right)-t_{i}^{*}$, contradicting the assumption that $\left(t_{1}^{*}, \ldots, t_{1}^{*}\right)$ is a Nash equilibrium. If $t^{*}>\hat{y}$, then let $i$ be any player for whom $t_{i}^{*}>0$. Define $\varepsilon \equiv \min \left\{t_{i}^{*}, t^{*}-\hat{y}_{i}\right\}$, positive because $\hat{y}_{i} \leq \hat{y}<t^{*}$, and consider $t_{i} \equiv t_{i}^{*}-\varepsilon \in\left[0, t_{i}^{*}\right)$, i.e., $\hat{y}_{i} \leq t_{-i}^{*}+t_{i}$ and $t_{-i}^{*}+t_{i}<t_{-i}^{*}+t_{i}^{*}$. By single peakedness, $v_{i}\left(t_{-i}^{*}+t_{i}\right)-\left(t_{-i}^{*}+t_{i}\right)>$ $v_{i}\left(t_{-i}^{*}+t_{i}^{*}\right)-\left(t_{-i}^{*}+t_{i}^{*}\right)$, i.e., $v_{i}\left(t_{-i}^{*}+t_{i}\right)+\omega_{i}-t_{i}>v_{i}\left(t_{-i}^{*}+t_{i}^{*}\right)+\omega_{i}-t_{i}^{*}$, contradicting the assumption that $\left(t_{1}^{*}, \ldots, t_{1}^{*}\right)$ is a Nash equilibrium. This proves (a).

To prove (b), let $i \notin T$ and $t_{i}^{*}>0$. Again, define $\varepsilon \equiv \min \left\{t_{i}^{*}, t^{*}-\hat{y}_{i}\right\}$, positive because $\hat{y}_{i}<\hat{y}=t^{*}$ ( by (a)), and proceed as in the previous paragraph.

Remark 2 The result is intuitive. Let $v_{i}(y)$ be differentiable with $v_{i}^{\prime \prime}(y)<0$ (or differentiable on $[0, \bar{y}]$ with $v_{i}^{\prime \prime}(y)<0$ on $[0, \bar{y})$ and $v_{i}^{\prime}(y)=0$ on $(\bar{y}, \infty)$. Then $b_{i}^{\prime}(y) \equiv v_{i}^{\prime}(y)-1$ is $i$ 's marginal net valuation of her contribution and $\hat{y}_{i}$ is defined by $b_{i}^{\prime}\left(\hat{y}_{i}\right) \leq 0$ with $b_{i}^{\prime}\left(\hat{y}_{i}\right) \hat{y}_{i}=0$. Let $\hat{y}>0$. If $i$ is a top contributor, then $b_{i}^{\prime}(\hat{y})=0$, whereas if not, then $b_{i}^{\prime}(\hat{y})<0$. But these are essentially the Kuhn-Tucker conditions of the Nash equilibrium.

\subsection{Single and multiple top valuators}

It follows from the Proposition that, in the single-top-valuator case (the set $T$ is a singleton), there is a unique Nash equilibrium. Provided that preferences are randomly selected among a rich enough set of quasilinear preferences, this is the typical situation. There is then a single contributor, and everybody else free rides.

If, on the contrary, and more exceptionally, we are in the multiple-top-valuator case, then the equilibrium contributions of top valuators are undetermined as long as they add up to $\hat{y}$. 


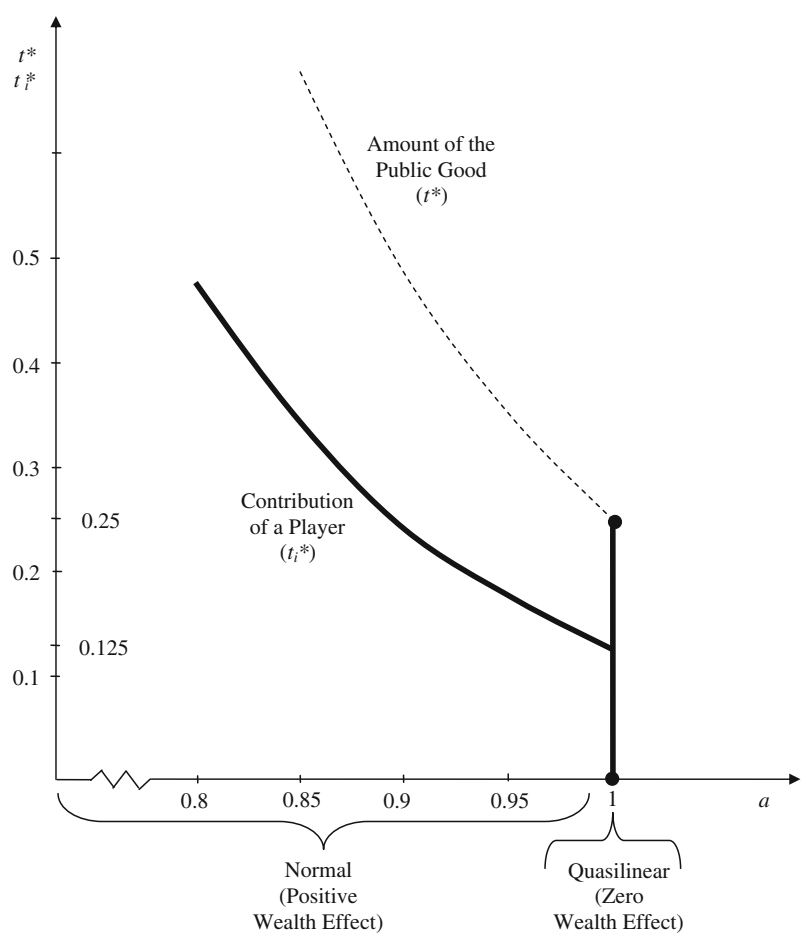

Fig. 1 The equilibrium correspondence as the wealth effect tends to zero. Example 1: Two players with identical preferences

\section{Comparison between normality and quasilinearity}

Quasilinear preferences are limits of preferences where both goods are normal as the wealth effects on the public good tend to zero. It is therefore instructive to compare the properties of the model with positive wealth effects, listed in Sect.3 above, with the corresponding ones in the limiting, quasilinear case.

(1) Uniqueness. The amount of the public good is uniquely determined both under normality and quasilinearity. But the equilibrium allocation, unique under normality, displays a degree of indeterminacy in the quasilinear, multiple-top-valuator case. This implies that, if we take a sequence of profiles of normal preferences that converge to a quasilinear, multiple-top-valuator profile of preferences, the equilibrium correspondence is not lower hemicontinuous. Example 1 illustrates: two identical individuals with $\omega_{i}=10$, and a sequence of utility functions of the form $\sqrt{y}+$ $m_{i}^{a}, i=1,2$, where the sequence of $a / \mathrm{s}$ is increasing and tends to one. Figure 1 graphs the equilibrium correspondence: the thick line indicates the contribution of one of the players, whereas the dotted line indicates the amount of the public good.

(2) Warr neutrality. In the single-top-valuator case, Warr neutrality trivially holds. In the multiple-top-valuator case, the set of equilibrium private-good consumptions is no longer invariant with respect to wealth redistributions among top valuators. But a version of Warr neutrality holds, namely that of Theorem 1 in 
Bergstrom et al. (1986), stating that after the redistribution, there is a new Nash equilibrium in which every player changes the amount of her contribution by precisely the change in her wealth.

\section{Free riding at positive wealth effects}

The form of free riding present in the quasilinear, single-top-valuator case, where all but one player contribute nothing, does not necessarily occur with normal preferences, possibly excepting large numbers of players or widely different preferences (see Andreoni 1988; Buchholz et al. 2006a). But it does typically occur when the wealth effects on the public good are positive but small.

Indeed, consider a sequence of differentiable, strictly quasiconcave normal economies converging to a quasilinear economy with a single top valuator. If $i$ is a non-top-valuator at the limit, then $b_{i}^{\prime}(y)<0$ there (Remark 2), and, because of the upper-hemicontinuity of the equilibrium correspondence (Fudenberg and Tirole 1991; see also Kohlberg and Mertens 1986), it must be the case that $\frac{\partial u_{i} / \partial y}{\partial u_{i} / \partial m_{i}}-1<0$ as the

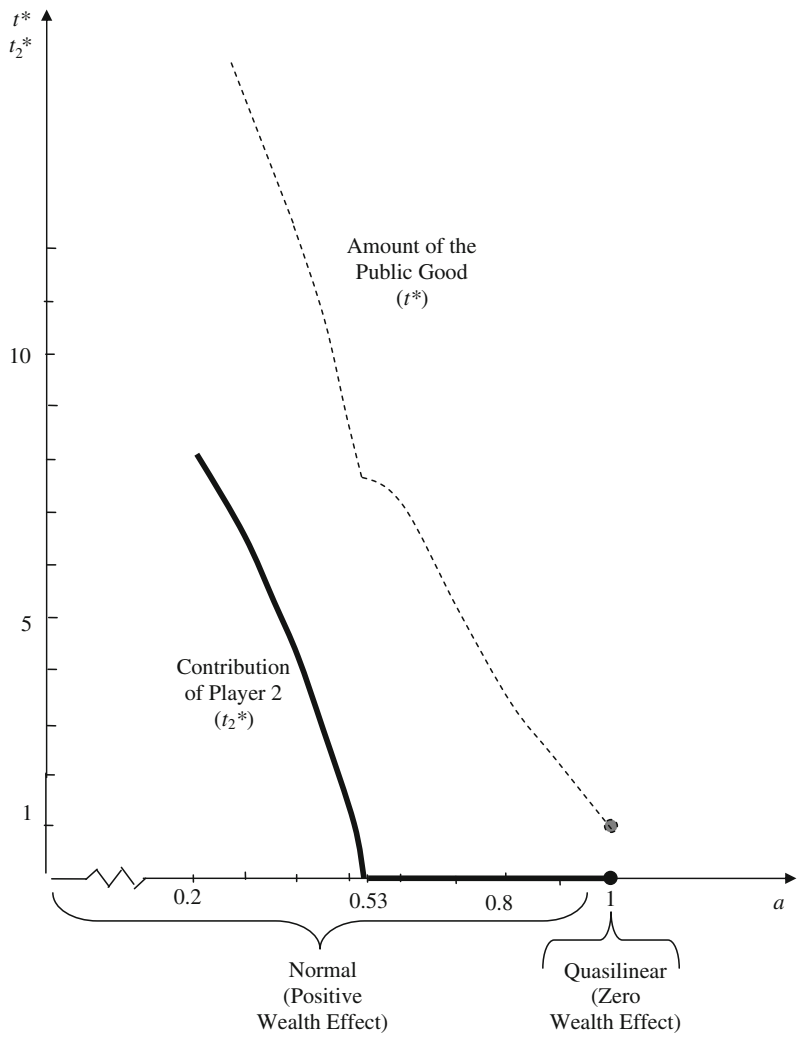

Fig. 2 The equilibrium correspondence as the wealth effect tends to zero: Example 2: Two players with different preferences 
sequence approaches its limit and wealth effects on the public good are positive but small. Hence, there will be a single contributor for small enough wealth effects.

This is a general observation, which applies to any number of players, and any nonzero degree of preference differentiation. Example 2 illustrates: two players, each endowed with 10 units of numeraire. Player 1's utility function is $2 \sqrt{y}+m_{1}^{a}$, and Player 2's is $\sqrt{y}+m_{2}^{a}$. Figure 2 depicts the equilibrium correspondence as $a$ tends to one: For high enough $a$ (in Example 2, for $a>0.53$ ), Player 1 is the only contributor, and Player 2 free rides.

\section{Conclusion}

When the wealth effects on the public good are small, as long as preferences are randomly selected, the presumption is that only one player contributes, whereas all the other players contribute nothing to the provision of the public good. The behavior of the non contributing players constitute "free riding" in the strong sense that the expression has in ordinary English usage. This result is independent from the number of players: it applies to both small and large numbers.

Open Access This article is distributed under the terms of the Creative Commons Attribution License which permits any use, distribution and reproduction in any medium, provided the original author(s) and source are credited.

\section{References}

Andreoni J (1988) Privately provided public goods in a large economy: the limits of altruism. J Public Econ 35:57-73

Bergstrom T, Blume L, Varian H (1986) On the private provision of public goods. J Public Econ 29:25-49

Bergstrom T, Blume L, Varian H (1992) Uniqueness of Nash equilibrium in private provision of public goods: an improved proof. J Public Econ 49:391-392

Buchanan JM (1964) What should economists do? South Econ J 30(3):213-222

Buchholz W, Cornes R, Peters W (2006a) On the frequency of interior Cournot-Nash equilibria in a public good economy. J Public Econ Theory 8(3):401-408

Buchholz W, Cornes R, Peters W (2006b) Lindahl vs. Cournot-Nash: the role of income distribution. FinanzArchiv 62(1):28-49

Fraser CD (1992) The uniqueness of Nash equilibrium in the private provision of public goods: an alternative proof. J Public Econ 49:389-390

Fudenberg D, Tirole J (1991) Game theory. MIT Press, Cambridge

Kohlberg E, Mertens J-F (1986) On the strategic stability of equilibria. Econometrica 54:1003-1038

Mas-Colell A, Whinston MD, Green JR (1995) Microeconomic theory. Oxford University Press, Oxford

Silvestre J (2008) The English language and economic terminology: an application to Catalan. In: Cabré MT, Bach C, Tebé C (eds) Literalidad y dinamicidad en el discurso económico, vol 21. Documenta Universitària, Sèrie Activitats 21, Barcelona

Warr P (1983) The private provision of a public good is independent of the distribution of income. Econ Lett 13:207-211 